\title{
Terapêutica nutricional para constipação intestinal em pacientes oncológicos com doença avançada em uso de opiáceos: revisão*
}

The nutritional therapy for bowel constipation in oncologic patients with advanced disease in opioids used: review

Helimar Senna dos Santos

\section{Resumo}

Constipação intestinal é um distúrbio comum em pacientes com câncer devido à síndrome de anorexia-caquexia, e sua freqüência está elevada naqueles com doença avançada e em uso de drogas opiáceas para tratamento da dor. A terapêutica nutricional na unidade de cuidados paliativos é fundamental para o sucesso do tratamento, pois ela minimiza os desconfortos causados por distúrbios como a constipação intestinal, oferece dietas adequadas ao estado fisiológico e proporciona prazer. A incorporação de nutrientes "laxativos" oferece benefícios, como a restauração do trânsito intestinal, aumento do peso das fezes e o restabelecimento da freqüência da defecação. Este artigo trata-se de uma revisão bibliográfica onde será abordada a constipação intestinal como conseqüência do uso de opióides para tratamento da dor em pacientes oncológicos em cuidados paliativos.

Palavras-chave: nutrição; dietoterapia; fibra na dieta; constipação; analgésicos opióides; pacientes incuráveis; cuidados paliativos.

\section{Abstract}

Bowel constipation is a typical disorder in cancer patients because of the anorexia-caquexia syndrome, and its frequency is high in patients with advanced disease who use opioids for pain management. The nutritional component in palliative care is very important for the overall treatment, as it helps lessening the severity of the di sorders the patient presents, making him feel better. The use of laxative nutrients are quite beneficial in reducing bowel constipation. This article is a review of the importance of nutrition for bowel constipation in palliative care patients who use opioids.

Key words: nutrition; diet therapy; dietary fiber; constipation; opioid analgesics; incurable patients; palliative care

\footnotetext{
*Artigo referente ao Trabalho de Conclusão do Curso de Nutrição O ncológica do Instituto N acional de Câncer. Professora do D epartamento de N utrição da Faculdade JK, Aluna Especial do Curso de M estrado em Ciências da Saúde da U niversidade de Braślia (UnB), Especialista em N utrição O ncológica pelo Instituto N acional de Câncer (IN CA), Especialista em N utrição Clínica e Cirúrgica pela FH DF (Programa de Residência) e Especialista em N utrição Clínica pela Universidade Federal Fluminense (UFF). Enviar correspondência para: SQ N 214 / 413 Bloco K, Asa N orte; 70873-110 Brasília, DF - Brasil. E-mail: helimarsenna@uol.com.br

Recebido em julho de 2001.
} 


\section{INTRODUÇÃO}

N o Brasil, o câncer é a segunda causa de morte por doença $\left(27,63 \%\right.$ do total). ${ }^{1} \mathrm{Em}$ 1999 , cerca de $1,8 \%$ dos gastos em alta complexidade destinaram-se para oncologia e $12,8 \%$ foram gastos com internação hospitalar para tratamento de câncer. ${ }^{2}$ Segundo a O rganização M undial de Saúde (O M S), a cada ano 9 milhões de pessoas morrem de câncer. Por isto, os programas de detecção precoce das neoplasias são fundamentais para o sucesso do tratamento e do bom prognóstico. ${ }^{3}$

Os avanços no campo da terapia oncológica tornaram muitos tumores curáveis ou passíveis de melhora. Q uando a cura não é possível, como em casos de pacientes que, na ocasião do diagnóstico, possuem doença avançada eincurável, o objetivo do tratamento é proporcionar uma qualidade de vida satisfatória, controlando a doença e seus sintomas com 0 uso de terapia paliativa. ${ }^{4-6}$ $N$ ela está incluído o alívio da dor destes pacientes, que ocorre em $67 \%$ dos pacientes com câncer metastático. ${ }^{7,8}$ Assim, 0 exercício da medicina paliativa, a qual se dedica ao estudo de pacientes com doença ativa, progressiva e avançada, para quem 0 prognóstico élimitado e a assistência évoltada para a qualidade de vida, passa a ser o principal objetivo do tratamento. ${ }^{9} 0$ controle da dor, de outros sintomas e dos problemas psicológicos, sociais e espirituais são soberanos. ${ }^{10}$

0 alívio da dor em pacientes oncológicos requer o uso de medicamentos, como os opióides, e de outras formas de terapia, como a neurocirurgia e anestesia. Entretanto, a administração de drogas opiáceas produz muitos efeitos colaterais, incluindo a constipação intestinal $(\mathrm{Cl})$, cujo controle se faz necessário para promover a qualidade de vida dos pacientes com doença avançada.

$\mathrm{A} \mathrm{Cl}$ é um problema comum entre os pacientes com doença avançada em uso de opiáceos e pode ser definida como a falta de freqüência ou a dificuldade de defecar, com os números de movimentos intestinais reduzidos, que resultam no desconforto ou dor, podendo ser diagnosticada como dois ou menos movimentos intestinais por semana.
Estudos têm demonstrado que cerca de $40 \%$ a $80 \%$ dos pacientes em cuidados paliativos cursam com constipação, e esta proporção aumenta para $90 \%$ ou mais quando os pacientes são tratados com opiáceos. Além deste fato, existe uma série de outros fatores implicados em sua gênese, como a baixa ingestão alimentar defibras, hipocalemia, falta de mobilidade, distúrbios neurológicos, uso de medicamentos e outros. Este distúrbio pode ser agravado ou precipitado com a administração de drogas opiáceas. ${ }^{11}$

Este artigo tem por objetivos identificar as principais terapias nutricionais anticonstipantes para os pacientes com câncer em cuidados paliativos, identificar os componentes dietéticos que auxiliam a regulação do trânsito intestinal e apontar as dificuldades da realização de uma al imentação equilibrada para o controle da $\mathrm{Cl}$, em estudos descritos na literatura nos últimos 15 anos.

\section{CONSTIPAÇÃO INTESTINAL COMO CONSEQÜÊNCIA DO USO DE OPIÁCEOS}

A dor do câncer é descrita como crônica e progressiva. Afeta a atividade, a função, 0 apetite, o sono dos pacientes, além de deprimir o paciente e aumentar o risco de suicídio. 8,12

Estima-se que $30 \%$ dos pacientes em tratamento curativo apresentam dor, e esta proporção aumenta para $70 \%$ a $90 \%$ dos pacientes, em caso de doença avançada. A dor deve ser aliviada para que o paciente volte a desenvolver sua capacidade funcional etenha uma sobrevida mais confortável até 0 momento final. Este alívio pode ser alcançado de muitas maneiras e o tratamento deve ser individualizado, com as abordagens necessárias para a minimização da dor.7,13,14

U ma das estratégias de tratamento da dor é o uso de analgésicos opióides, que são compostos naturais ou sintéticos que produzem efeitos semelhantes aos da morfina. São fármacos obtidos do extrato Papaver somniferum - papoula, como a morfina e a codeína. ${ }^{15,16} \mathrm{O}$ s principais medicamentos opióides são: a) morfina (utilizada amplamente), b) meperidina (opiáceo 
sintético, não relacionado à morfina), c) metadona (opiáceo sintético, tão potente quanto a morfina), d) fentanil (quimicamente relacionado à meperidina, é 80 vezes mais potente que a morfina), e) heroína, f) propoxifeno, g) codeína (menos potente que a morfina, mas potente por via oral), h) pentazocina, i) buprenorfina, j) naloxona e; I) naltrexona1. ${ }^{5}$ Existem também os analgésicos não opióides, como 0 ácido acetilsalicílico e antiinflamatórios não esteróides (AINE) que são importantes no tratamento da dor, pois se verifica uma alta concentração de prostaglandinas no local da dor que pode ser bloqueada pelos AINE. ${ }^{7}$

A morfina e seus correlatos combinamse seletivamente a vários sítios de reconhecimento existentes no organismo para produzirem os efeitos farmacológicos. ${ }^{17} \mathrm{OS}$ locais no cérebro envolvidos na transmissão da dor e na alteração da reatividade aos estímulos nociceptivos (dolorosos) parecem ser os principais sítios nos quais os opióides agem. O s receptores também podem ser encontrados, em altas concentrações, no trato gastrointestinal (TGI). ${ }^{15,16}$

N o T GI, quando os terminais são ligados com opióides, ocorre redução no estímulo que cria necessária contração propulsiva. Esta ação reduz a motilidade gástrica, as secreções biliar, pancreática e intestinal, e atrasa a digestão dos alimentos. Este atraso expõe 0 conteúdo intestinal à superfície mucosa por um longo tempo, aumentando a absorção de fluidos. ${ }^{18} 0$ longo tempo de trânsito intestinal e a absorção de grande quantidade de fluidos tornam as fezes ressecadas e endurecidas causando a $\mathrm{Cl}$. As dificuldades e desconfortos relacionados à $\mathrm{Cl}$ podem estar associados à sensação de esvaziamento retal incompleto, sucedido por dor abdominal, flatulência, distensão, anorexia, cefaléia, edema e a presença ou não de náuseas. 19-21

A constipação, como consequência do uso de opiáceos, é um efeito reconhecido e inevitável. O s efeitos constipantes são mediados por uma ação no sistema nervoso central e local. No estômago, a motilidade pode estar reduzida, mas o tônus deve aumentar, principalmente na porção central e a secreção de ácido clorídrico está reduzida. $\mathrm{N} o$ intestino delgado, o tônus de repouso aumenta, com espasmos periódicos, mas a amplitude das contrações não propulsivas estão diminuídas, isto atrasa a passagem do bolo fecal e permite uma absorção maior de água, resultando na constipação. ${ }^{17,18,23} \mathrm{Em}$ 1946 , foi realizado um estudo que mensurou os efeitos da morfina sobre o aumento da motilidade do tipo I (descrita como os movimentos segmentados) e redução dos movimentos tipo II (movimentos que conduzem a evacuação), resultando em contato prolongado dos alimentos com a parede intestinal e aumento da absorção de fluidos. Além disto, foi verificado que a morfina atrasa 0 trânsito intestinal, especificamente no ceco e no cólon ascendente, sugerindo que os problemas de propulsão se iniciam no cólon proximal e culminam distalmente com a inibição da defecação. ${ }^{24}$

Este distúrbio ocorre por razões variadas, que vão desde o estilo de vida até condições patológicas. Os pacientes com câncer avançado desenvolvem falência autonômica, a qual está associada a síndrome anorexiacaquexia, relacionada com a motilidade gastrointestinal anormal e sintomas como a anorexia, náuseas e constipação. ${ }^{18,22}$

A avaliação da $\mathrm{Cl}$ deve ser minuciosa, uma vez que estes pacientes possuem vários fatores de risco e as complicações devem ser avaliadas cuidadosamente. Devem ser considerados a história da freqüência e da dificuldade de defecação, a história de dor ou tenesmo, os sintomas relacionados à constipação e exames físico e retal para verificar a presença de sangramentos. ${ }^{20,22}$ D eve-se utilizar escalas eficazes que medem a presença e a severidade da constipação, adaptando-as às populações em que serão aplicadas.

0 controle da dor é essencial para a boa prática em saúde e requer cuidadosa consideração acerca da dose apropriada, tipo de droga e doença a ser tratada. A dor associada ao câncer ou outras doenças avançadas deve ser tratada, e preocupações com tolerância e dependência devem ser ignoradas, quando necessário, em favor do conforto dispensado ao paciente, entretanto a dose limite da droga deve ser sempre respeitada, para evitar 0 desenvolvimento de 
tolerância e alterações intestinais, como a constipação. ${ }^{17}$

\section{TERAPÊUTICA NUTRICIONAL}

A alta freqüência da $\mathrm{Cl}$ em cuidados paliativos indica que a profilaxia deve ser aplicada, uma vez que sua causa é multifatorial. ${ }^{23} \mathrm{Em}$ se tratando de $\mathrm{Cl}$ induzida pelo uso de narcóticos, o tratamento deve ser preventivo. ${ }^{24}$

A mudança no estilo de vida dos pacientes deve ser recomendada e é benéfica por melhorar as funções fisiológicas básicas. $0 \mathrm{~s}$ pacientes devem: a) possuir um tratamento individualizado; b) ser orientados quanto aos malefícios do uso excessivo de laxantes; c) ser encorajados a ter hábitos intestinais regulares, respeitando, sempre que possível, o reflexo gastrocólico após as refeições, principalmente após o desjejum. ${ }^{25,26}$

Segundo a Associação D ietética Americana (AD A), o controle da constipação pode ser realizado através do consumo de fibras (25 a 35g/dia, para indivíduos com mais de 20 anos e de 10 a $13 \mathrm{~g}$ por $1000 \mathrm{Kcal}$ para idosos), da incorporação de dieta balanceada, isto é, com altas quantidades de carboidratos complexose reduzida quantidade degorduras, da atividade física regular e da ingestão hídrica adequada. 27,28

0 s benefícios gastrointestinais reconhecidos com o uso de fibras dietéticas (FD ) são: a) efeito laxativo, promovido pelas fibras insolúveis; b) redução na pressão intral uminal, pois o bolo fecal se torna mais macio e úmido; c) redução do tempo de trânsito intestinal, protegendo 0 cólon de substâncias citotóxicas; d) elevação do volume fecal e; e) proliferação bacteriana el evada ${ }^{29}$. Contudo, estes ben efícios não são efetivos sem que haja um consumo adequado de líquidos, pois eles são importantes para lubrificar e aumentar o processo de mistura das fezes. É recomendado que seja ingerido, por dia, de um e meio a dois litros de água (1,5 a 2L/dia). Caso ocorra um baixo consumo hídrico, o paciente poderá apresentar efeitos adversos causados pelo consumo de fibras, entre estes podemos observar desde a produção excessiva de flatos até obstrução em qualquer parte do tubo digestivo, sendo mais comum nos intestinos. Para os pacientes que apresentam aumento da plenitude gástrica e por isto possuem dificuldades para ingerir os alimentos e a quantidade hídrica recomendada, algumas medidas dietoterápicas como: a) administração de líquidos com temperaturas quentes meia hora antes de o paciente evacuar; b) oferta hídrica na forma de gelatinas; c) preparações líquidas com valores nutricionais adequados e; d) evitar a oferta de líquidos que contenham poucos nutrientes necessários, ${ }^{24,30}$ são indicadas para promover o consumo de líquidos adequado, sem que ocorram prejuízos no consumo de nutrientes.

Em pacientes com doença avançada, 0 tratamento da constipação é baseado na prevenção, através da educação nutricional, possibilitando-Ihes o conhecimento do funcionamento intestinal e de medidas preventivas adequadas, como o consumo de pelo menos 5 a $10 \mathrm{~g}$ de fibras por dia. Q uando o distúrbio já está instalado, a dieta e o uso de agentes laxativos naturais como a lactulose, que embora seja um dissacarídeo sintético formado por frutose e galactose, promove um efeito laxativo, uma vez que não é metabolizada ou absorvida no organismo, podem fazer parte do tratamento de escolha. ${ }^{24,31} \mathrm{Um}$ outro nutriente que tem apresentado resultados satisfatórios em pacientes com constipação crônica, é o isomalto-oligossacarídeo, que é um amido resistente à digestão humana. A suplementação diária com $10 \mathrm{~g}$ por 30 dias em pacientes do sexo masculino, promoveu a melhora da freqüência da defecação e da sensação de tenesmo, além de aumentar produção de ácidos graxos de cadeia curta e a absorção intestinal de cálcio e fósforo. ${ }^{32}$

A modificação física daFD também é uma medida dietética que possibilita a ingestão de quantidades suficientes destes produtos sem que ocorra 0 aumento substancial da plenitude e como conseqüência, menor ingestão de alimentos. 0 cozimento dos alimentos pode causar quebra das reações, que, aparentemente, aumentam o conteúdo de fibras dos alimentos. Isto ocorre porque há uma perda de água dos alimentos durante a cocção, tornando a FD mais biodisponível. ${ }^{33}$

O utra alternativa para o tratamento da $\mathrm{Cl}$ 
é a suplementação de L-arginina, que reduz 0 atraso no trânsito intestinal produzido pela morfina, podendo ser um efeito causado pelo aumento da liberação de óxido nítrico, que tem sido identificado como neuro-modulador intestinal. ${ }^{24}$ Porém deve-se observar que 0 óxido nítrico é um radical livre que causa efeitos deletérios ao organismo e pode resultar em complicações indesejáveis como toxicidades cardíaca, renal, pulmonar entre outras. Seu uso deve vir sempre acompanhado de suplementações de vitaminas antioxidantes, como as vitaminas $C, A$ e E.

O sucesso do tratamento nestes pacientes é dificultado pela baixa ingestão de alimentos, devido a causas variadas pertinentes à própria doença avançada, e também por causa das alterações que a constipação pode causar, como náuseas, vômitos, sensação de plenitude e anorexia. Estes fatores são cruciais no tratamento dietoterápico, pois limitam a prática do profissional nutricionista que atua em unidades detratamento paliativo. 0 plano dietoterápico destes pacientes deve ser o mais individualizado possível e promover 0 efeito laxativo desejado. As refeições devem ser fracionadas, sendo oferecidas na consistência e temperatura que melhor forem convenientes ao paciente. Elas devem ser compostas por alimentos de hábito do paciente e com aspecto e sabor agradáveis.

0 tratamento realizado na enfermaria de cuidados paliativos deve possuir a integração de toda a equipe hospitalar a fim de controlar todas as causas possíveis de $\mathrm{Cl}$. A conduta nutricional dependerá do momento e dos sintomas encontrados, isto é, deverá ser avaliada a cronicidade da $\mathrm{Cl}$ e 0 grau das complicações existentes. A terapêutica nutricional deve ser adequada às condições atuais, levando em consideração a capacidade gástrica do paciente, com a oferta de quantidades satisfatórias de calorias, fibras e líquidos, suficientes para amenizar os sintomas da $\mathrm{Cl}$.

\section{CONCLUSÃO}

A dietoterapia ou terapêutica nutricional é parte fundamental no tratamento e na qualidade de vida de pacientes oncológicos em cuidados paliativos, uma vez que um dos prazeres que ainda pode estar conservado é 0 consumo de alimentos.

A constipação intestinal como conseqüência do uso de opióides é um sintoma comum e interfere na qualidade de vida dos pacientes. Seu controle está, principalmente, na intervenção nutricional adequada, permitindo que o distúrbio seja minimizado sem que métodos invasivos e desconfortáveis sejam utilizados. A alimentação deste pacientes deve ser compatível com seu estado fisiológico atual. Assim, a ingestão hídrica e a oferta de fibras e de nutrientes devem ser realizadas de acordo com a capacidade de aceitação do paciente, isto é, deve-se evitar a ocorrência de plenitude gástrica e outros desconfortos físicos e psicológicos. É importante que o bem estar do paciente seja a prioridade no momento em que a terapia, seja ela nutricional ou não, for instituída.

Alguns componentes dietéticos como a lactulose, o isomalto-oligossacarídeo, a FD e a $L$-arginina exercem efeitos positivos no intestino, produzindo a melhora da eficiência dos movimentos intestinais, o aumento do peso das fezes e a elevação da freqüência da defecação. Estes nutrientes devem integrar a terapia nutricional paliativa, pois minimizam os sintomas de $\mathrm{Cl}$ e conseqüentemente restauram o bem-estar do paciente. $M$ edidas dietéticas eficazes, como a oferta hídrica satisfatória e a ingestão adequada de nutrientes auxiliam no tratamento da $\mathrm{Cl}$ e possibilitam a manutenção do estado nutricional do paciente sem que ocorra depleção maior. A dieta deve ser atrativa e composta por alimentos de hábito dos pacientes.

É importante também avaliar a $\mathrm{Cl}$ e diferenciá-la de outros distúrbios intestinais que possam vir a ocorrer, como a impactação fecal. É necessário conhecer o momento em que o tratamento com opiáceos foi instituído e se houve terapias dietéticas ou outras medidas terapêuticas aliadas a este. U m acompanhamento eficaz, tanto a nível ambulatorial, quanto na enfermaria é importante para a determinação da terapia nutricional a ser instituída e para conhecer 
os sintomas iniciais apresentados pelos pacientes.

Em cuidados paliativos, todos os efforços devem estar direcionados à promoção do bemestar físico e psicológico dos pacientes. 0 profissional nutricionista deve ter em mente que o consumo de alimentos é capaz de minimizar desconfortos, cuidar de distúrbios orgânicos, nutrir e proporcionar prazer.

\section{REFERÊNCIAS BIBLIOGRÁFICAS}

1. Instituto $\mathrm{N}$ acional de $\mathrm{C}$ âncer (Brasil). Estimativa da incidência e mortalidade por câncer no Brasil 1999. Rio deJ aneiro; 2000.

2. Secretaria $\mathrm{N}$ acional de Assistência à Saúde (Brasil). Assistência H ospitalar no SU S: 1995/ 1999. Brasília; 2000. 392p.

3. Instituto $\mathrm{N}$ acional de $\mathrm{C}$ âncer (Brasil). Controle do câncer: uma proposta de integração ensinoserviço. 3a ed. Rio de Janeiro: IN CA; 1999.

4. Andreoli TE, BennettJC, Carpenter CCJ, Plum $F$, Smith LH J r. Cecil medicina interna básica. 4a ed. Rio de Janeiro: Guanabara Koogan, 1999.

5. Sasse A. Câncer. Disponível em: http:// sites.mpc.com.br/andre.sasse/cancer.htm. p. 1-3.

6. O ttery F. Cancer and cachexia implicationsfor care of thecancer patient. Philadelphia; 1996.

7. Instituto $\mathrm{N}$ acional de $\mathrm{C}$ âncer (Brasil). Coordenação de Programas de Controle de Câncer. $O$ alívio da dor do câncer. Rio de Janeiro: IN CA; 1997.

8. Chang H M . C ancer pain management. M ed Clin N orth America 1999;83(3):711-36.

9. Oxford U niversity. $O$ xford texbook of palliativemedicine. 2nd ed. N ew York: O xford M edical Publications; 1998.

10. World $\mathrm{H}$ ealth $\mathrm{O}$ rganization. Cancer pain relief and palliative care. Genebra;1996. vol.11 (Technical Report Series; no 804).

11. D aeninck PJ, BrueraE. Reduction in constipation and laxativerequirementsfollowing opioid rotation to methadone: a report of four cases. I Pain Symptom M anage 1999;18(4):303-9.

12. LazarusH , Fitzmartin RD, G oldenheim PD . A multi-investigator clinical evaluation of oral controlled-releasemorphine(M S Contin tablets) administered to cancer patients. H osp J 1990;6(4):1-15.

13. Viganó A, Bruera E, Suarez-Almazor M E. Age, pain intensity, and opioid dose in patients with advanced cancer. Cancer 1998;83(6):124450.

14. H anks GW. C ancer pain and theimportance of its control. Anticancer D rugs 1995;6(Suppl 3) 14-7.

15. H arvey RA, Champe PC. Farmacologia ilustrada. 2a ed. Artmed; 1998.

16. ZyliczZ, TwycrossRG . O ral opioidsin thetreatment of cancer pain. N eth J M ed 1991;39(1):108-14.

17. Katzung BG . Farmacologia básica e clínica. 5a ed. Rio deJ aneiro: G uanabara Koogan; 1992.

18. Canty SL. Constipation asa side effect of opioids. O ncol N ursForum 1994;21(4):739-45.

19. Sykes N P. Current approaches to the management of constipation. Cancer Surv 1994;21:137-46.

20. M ancini I, BrueraE. Constipation in advanced cancer patients. Support $\mathrm{C}$ are $\mathrm{C}$ ancer 1998;6(4):356-64.

21. Cancer Information Service. Constipation, impaction, and bowel obstruction (PDQ ${ }^{\circledR}$ ) supportive care - health professionals. Available from: http://www.cancernet.nci.nih.gov/cgibin/srch.

22. Alessi CA, H enderson CT. Constipation and fecal impactation in thelong-term carepatient. Clin G eriatr M ed 1988;4(3):571-88.

23. SykesN P.T Thetreatment of morfine induced constipation. EurJ Palliat C are 1998;5(1):12-5.

24. Cameron JC. Constipation related to narcotic therapy. Cancer N urs 1992;15(5):372-7.

25. Sweeney M . C onstipation: diagnosis and treatment. H omeC are Provid 1997;2(5):250-5.

26. Wilson JA. Constipation in the elderly. Clin Geriatr M ed 1999;15(3):499-510.

27. Gorman M A, Bowman C. Position of the American D ietetic Association: health implica tions of dietary fiber. J Am Diet Assoc 1993;93(12):1446-7.

28. M arlettJA, Slavin JL. Position of theAmerican Dietetic Association: health implication sof dietary fiber. J Am D iet Assoc 1997;97(10):11579.

29. D avidson $M H$, M cdonald A. Fiber: forms and functions. N utr Res 1998;18(4):617-24.

30. K ritchevsky D. Dietary fiber. Ann Rev N utr 1988;8:301-28.

31. Clausen M R, M ortensen PB. Lactulose, dissacharides and colonic flora. D rugs 1997;53(6):930-42. 
32. Chen H L, Lu YH, Lin JJ, Ko LY. Effects of isomalto-oligosaccharides on bowel functions and indicators of nutritional status in constipated elderly men. J Am Coll Nutr
2001;20(1):44-9.

33. Slavin JL. D ietary fiber: classification, chemical analyses, and food sources. J Am Diet Assoc 1987;87(9):1164-71. 